\title{
A dosimetric phantom study of thoracic radiotherapy based on three-dimensional modeling of mediastinal lymph nodes
}

\author{
JI-BIN ZHANG ${ }^{1}$, LI-RONG ZHAO ${ }^{1}$, TIAN-XIANG CUI ${ }^{1}$, XIE-WAN CHEN ${ }^{2}$, QIAO YANG ${ }^{1}$, \\ YI-BING ZHOU ${ }^{1}$, ZHENG-TANG CHEN ${ }^{1}$, SHAO-XIANG ZHANG ${ }^{3}$ and JIAN-GUO SUN ${ }^{1}$ \\ ${ }^{1}$ Cancer Institute of The People's Liberation Army, Xinqiao Hospital, Third Military Medical University, \\ Chongqing 400037; Departments of ${ }^{2}$ Medical English and ${ }^{3}$ Digital Medicine, College of Basic Medicine, \\ Third Military Medical University, Chongqing 400038, P.R. China
}

Received June 12, 2017; Accepted November 16, 2017

DOI: $10.3892 / \mathrm{ol} .2018 .8084$

\begin{abstract}
The aim of the present study was to investigate the optimal strategy and dosimetric measurement of thoracic radiotherapy based on three-dimensional (3D) modeling of mediastinal lymph nodes (MLNs). A 3D model of MLNs was constructed from a Chinese Visible Human female dataset. Image registration and fusion between reconstructed MLNs and original chest computed tomography (CT) images was conducted in the Eclipse ${ }^{\mathrm{TM}}$ treatment planning system (TPS). There were three plans, including 3D conformal radiotherapy (3D-CRT), intensity-modulated radiotherapy (IMRT) and volumetric-modulated arc therapy (VMAT), which were designed based on 10 cases of simulated lung lesions (SLLs) and MLNs. The quality of these plans was evaluated via examining indexes, including conformity index (CI), homogeneity index and clinical target volume (CTV) coverage. Dose-volume histogram analysis was performed on SLL, MLNs and organs at risk (OARs). A Chengdu Dosimetric Phantom (CDP) was then drilled at specific MLNs according to 20 patients with thoracic tumors and of a medium-build. These plans were repeated on fused MLNs and CDP CT images in the Eclipse ${ }^{\mathrm{TM}}$ TPS. Radiation doses at the SLLs and MLNs of the CDP were measured and compared with calculated doses. The established 3D MLN model demonstrated the spatial location of MLNs and adjacent structures. Precise image registration and fusion were conducted between reconstructed MLNs and the original chest CT or CDP CT images. IMRT demonstrated greater values in CI, CTV coverage and OAR (lungs and spinal cord) protection, compared with 3D-CRT and VMAT $(\mathrm{P}<0.05)$. The
\end{abstract}

Correspondence to: Professor Jian-Guo Sun, Cancer Institute of The People's Liberation Army, Xinqiao Hospital, Third Military Medical University, 83 Xinqiao Street, Chongqing 400037, P.R. China E-mail: sunjg09@aliyun.com

Key words: mediastinal lymph nodes, three-dimensional reconstruction, radiation phantom, plan optimization, dosimetric measurement, thoracic radiotherapy deviation between the measured and calculated doses was within $\pm 10 \%$ at SLL, and at the $2 \mathrm{R}$ and 7 th MLN stations. In conclusion, the 3D MLN model can benefit plan optimization and dosimetric measurement of thoracic radiotherapy, and when combined with CDP, it may provide a tool for clinical dosimetric monitoring.

\section{Introduction}

Thoracic malignancies are the most common types of cancer globally $(1,2)$. One of the primary treatments for this type of malignancy is radiotherapy (3). It is known that the fundamental principle of radiotherapy is to deliver an accurate therapeutic dose to the tumor tissue and avoid excessive radiation exposure to the adjacent normal tissues, thus increasing local tumor control probability (TCP) and decreasing normal tissue complication probability (NTCP) (4). Conventionally, thoracic radiotherapy is planned using three-dimensional conformal radiotherapy (3D-CRT) and intensity-modulated radiation therapy (IMRT) (3). Recently, volumetric-modulated arc therapy (VMAT) has become a focus of studies worldwide due to its increased delivery efficiency over 3D-CRT and IMRT (5); however, a number of studies have reported controversial findings on the conformity of targets and the protection of healthy structures in thoracic radiotherapy (6-8). This can be attributed to interpatient diversity and the contrasting definition of the target in different radiotherapy centers. The evaluation and comparison of the quality and efficacy of distinct radiotherapy strategies remains challenging (9).

Staging of involved mediastinal lymph nodes (MLNs) serves a function in determining the treatment strategy and overall patient prognosis (10). In 1996, the American Thoracic Society and American Joint Committee for Cancer (AJCC) proposed a criterion to divide intrathoracic lymph nodes (ITLN) into groups, nine categorized as MLNs and the other five within the hilus and lobe of the lung group (11). The Union for International Cancer Control and the International Association for the Study of Lung Cancer also support this classification of ITLN $(12,13)$; however, the method to clearly recognize and localize MLNs, and to distinguish them from adjacent structures remains a clinical problem. In a clinical 
setting, current identification of MLNs primarily involves 2D white-gray imaging, including computed tomography (CT), magnetic resonance imaging (MRI) and positron emission tomography-CT, which is inefficient at distinguishing MLNs from blood vessels, muscles and soft tissues (14). The Chinese Visible Human $(\mathrm{CVH})$ dataset has been established by Third Military Medical University (Chongqing, China) $(15,16)$; thus, 3D reconstruction of high-quality MLN images and thoracic structures has become possible. Images of $\mathrm{CVH}$ dataset sections were captured in high structural and spatial resolutions, and $\mathrm{CVH}$ images demonstrated advantages, including high resolution, lack of deformation, high articulation and thinness of section, compared with medical images. Thus, the CVH dataset has been frequently used as a tool to construct 3D medical models to discern miniscule structures; for example, 3D thorax models have been reconstructed based on the CHV1 dataset as a learning tool for interpreting human thoracic anatomy and virtual thoracic and cardiovascular surgery for medical students and junior surgeons (17). Furthermore, the $\mathrm{CVH}$ head dataset has been used as a brain atlas for locating the subthalamic nucleus prior to deep brain stimulation surgery (18). However, no 3D MLN models or similar tools have been used in previous studies, to the best of our knowledge.

It has been previously demonstrated that the modern treatment planning system (TPS) is able to accurately predict the patient dose (19); however, the actual absorbed doses generally deviate from calculated doses in a certain range. The accurate absorbed doses of regions of interest (ROIs), including planning target volume (PTV) and organs at risk (OARs), cannot be obtained from a patient receiving radiotherapy $(20,21)$. Therefore, a standard method is required to measure the in vivo radiation doses, which may be explored by a simulation. The present study attempts to improve the plan evaluation and dosimetric measurement using a constructed visual model.

In the present study, a 3D MLN digital model was constructed based on the CVH dataset to use as a virtual tool to assist in obtaining improved contours of lymph nodes, and to optimize the plan in TPS and dosimetric measurement of the Chengdu Dosimetric Phantom (CDP), a state-of-the-art heterogeneous phantom (22). The CDP is a novel radiation phantom of a person of medium-build and Chinese origin, with a height of $170 \mathrm{~cm}$ and a weight of $65 \mathrm{~kg}$ (22). CDP was named by the International Commission of Radiation Units and Measurements (report 48) and has been accepted internationally (23). Similar to other radiation phantoms, the CDP possesses a humanoid shape (24). The CDP is composed of the material with the same ratio of atomic elements as real human bodies (25); therefore, it is bioequivalent with human beings in irradiation, energy transfer and radiation distribution, and is regarded as an avatar to assess damage in reality (25). All of these characteristics of the CDP can be combined with a 3D MLN model for dosimetric measurement in thoracic radiotherapy.

The hypothesis of the present study was that the $\mathrm{CVH}$ dataset and CDP phantom could be combined to build a 3D MLN model, which could be used to test the quality of thoracic radiotherapy plans. The results may provide implications for clinical practice.

\section{Materials and methods}

Image segmentation of the CVH dataset. The CVH female dataset (CVH2), available at http://www.chinesevisiblehuman .com, was selected for the $3 \mathrm{D}$ reconstruction of MLNs. The CVH2 cadaver was 22 years old at mortality, $162 \mathrm{~cm}$ in height, $54 \mathrm{~kg}$ in weight and free of organic lesions. The subject was sectioned in axial planes, and high-resolution anatomic images were acquired. The complete series of anatomical images constitute the $\mathrm{CVH} 2$, with each slice $0.5 \mathrm{~mm}$ in thickness and a resolution of $3,072 \times 2,048$ pixels (16). The high-resolution cryosectional color photographic images were captured using a high-definition digital camera. A total of 380 images of the mediastinum (Fig. 1A) were imported into the Photoshop CS software (version 3.0; Adobe Systems, Inc., Sam Jose, CA, USA) for editing, including contouring MLN stations. An expert anatomist and a physician contoured and segmented the 1st-8th MLN stations on every layer manually, ranging from the sternum to the thoracic vertebrae and from the supraclavicular area to the bottom of the lungs. The adjacent MLN structures were also contoured, including the esophagus, trachea, left and right bronchi, thymus, sternum, thoracic duct and vertebrae, spinal cord, thoracic great vessels and heart. Every structure was then awarded different red, green and blue values and filled with different colors (Fig. 1B). The layers were established and input one by one in order to complete the $3 \mathrm{D}$ reconstruction.

Surface and volume reconstructions. Surface and volume reconstructions were performed as previously described (17). Briefly, the original images in PSD format were converted to PNG format with Photoshop CS. The color images were converted to grayscale mode and imported into the Amira program (version 5.2.2; Thermo Fisher Scientific, Inc., Waltham, MA, USA). Threshold segmentation was used to extract the data of different anatomical structures, and then surface reconstruction was applied to gain the surface model of these anatomical structures. As for volume reconstruction, the original sectional images in PNG format were cut appropriately by Photoshop CS and imported into the Amira program. According to the 'Orthoslice' and 'Oblique Slice' commands, random sections were acquired through virtual cutting, including coronal and sagittal planes, or in any angle or layer of the target structures. MATLAB software (version 8.1; MathWorks, Natick, MA, USA) was used to convert CVH images into the Digital Imaging and Communications in Medicine (DICOM) format.

Image registration and fusion in TPS. The $\mathrm{CVH} 2$ cadaver underwent imaging acquisition procedures via a $\mathrm{CT}$ scan and standard radiological imaging. Chest CT images were also obtained. The chest CT images and MLN images in DICOM format were imported to the Eclipse ${ }^{\mathrm{TM}}$ TPS (version 8.3; Varian Medical System. Inc., Palo Alto, CA, USA). Rigid alignment was performed following the selection of four points on the bony landmarks. The procedure was repeated automatically three to five times in order to obtain the best fused images.

Formulation of the plan for thoracic radiotherapy in a $3 D$ MLN model. Fused images were transferred into Eclipse TPS for radiotherapy plan formulation. One virtual simulated lung 

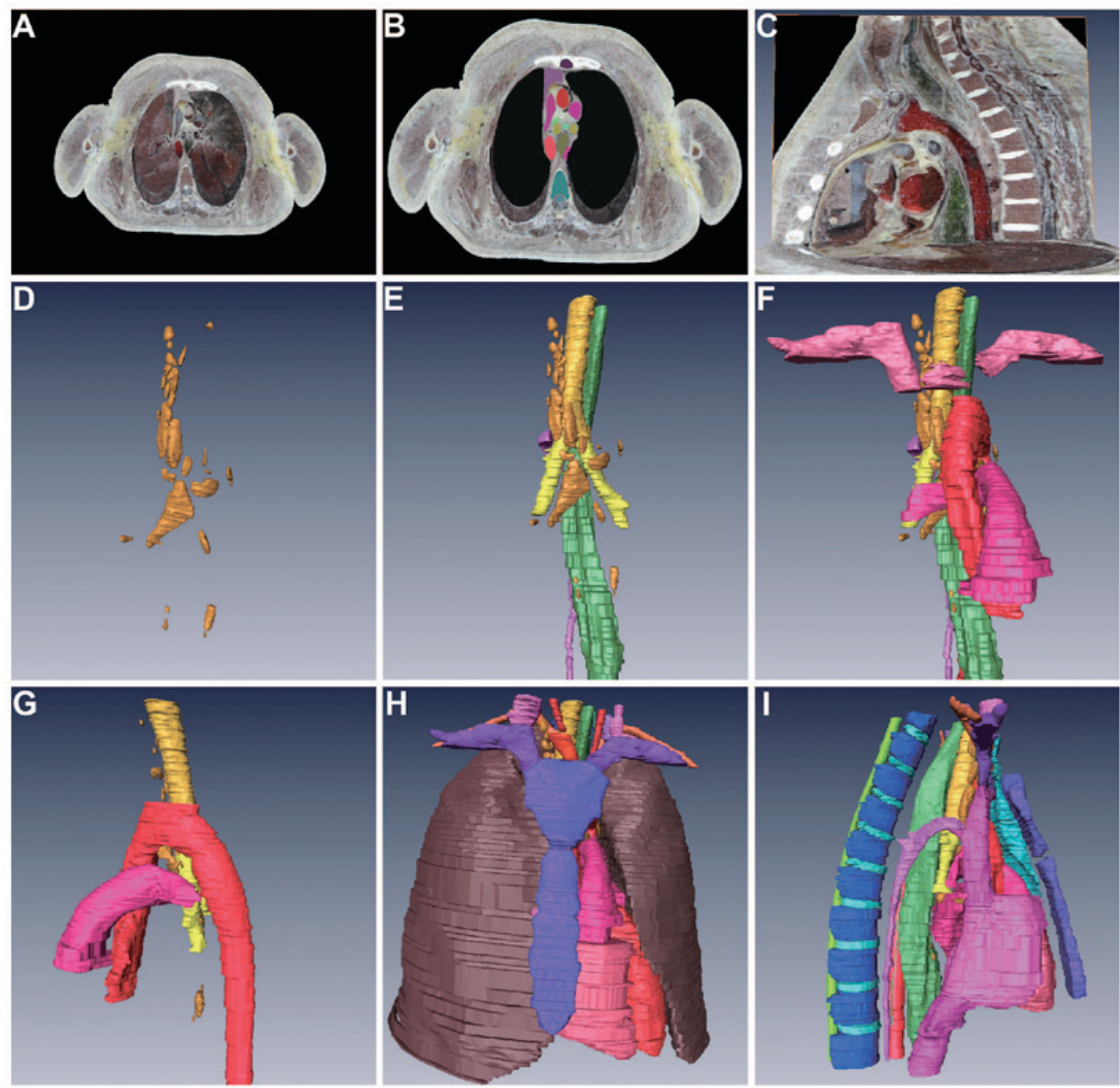

Figure 1. 3D reconstruction of MLNs and adjacent structures. (A) The original sectional image (2,085th layer). (B) Image segmentation of the transverse-sectional plane. (C) Volume reconstruction of the sagittal-sectional plane. (D) 3D model of the MLNs alone. (E) 3D model of eight MLN stations, esophagus and trachea. (F) Front view of the 3D model of MLNs and adjacent structures. (G) Right view of the 3D model of MLNs and adjacent structures. (H) Front view of the 3D model of structures adjacent to MLNs. (I) Left view of the 3D model of structures adjacent to MLNs. MLNs, mediastinal lymph nodes; 3D, three-dimensional.

lesion (SLL) was produced for each case in a 3D MLN model in Eclipse TPS. A total of 10 cases of SLL were assigned at different spots of the right lung. Gross tumor volume (GTVt), lymph nodes at the 2nd, 4th and 7th stations (GTVn) and OARs (lungs, heart and spinal cord) were contoured on the fused images. Planning GTVt (PGTV) and clinical target volume (CTV) were obtained by 3-mm and 8-mm 3D enlargement of the GTVt and GTVn, respectively. A dose of $66 \mathrm{~Gy} / 33 \mathrm{~F}$ was administered to the CTV. The radiotherapy plan was created for each case by three distinct technologies, including 3D-CRT with three fields, IMRT with five fields and VMAT with a full arc (range, $181-179^{\circ}$ ) based on the anisotropic analytical algorithm. The VMAT fields used a dynamic multi-leaf collimator, and variable dose rates and gantry speeds. Plan optimization was performed with dose volume objectives in IMRT and a progressive resolution optimizer in VMAT. Each plan should be created and confirmed by three different radiotherapy physicists and accepted by three different radiotherapy physicians. Dosimetric parameters in dose-volume histograms (DVHs) are presented as the mean \pm standard deviation (SD).
Drilling the CDP. To drill the MLN checkpoints onto the CDP model, three distances were measured in the CT images of patients. To avoid deviation in drilling the CDP due to different sizes of female breasts, male patients were used to build the model for a female dataset. Male patients had similar characteristics to those of the medium CDP $(170 \mathrm{~cm}$ in height and $65 \mathrm{~kg}$ in weight), whose thoracic tumors were selected to measure $\mathrm{X}, \mathrm{Y}$ and $\mathrm{Z}$ distances. In the plane of the coronal section, the vertical distance $(\mathrm{X})$ was measured from the central point of specific MLNs to the middle line. In the plane of the transverse section, the vertical distance (Y) was measured from the central point of specific MLNs to the surface skin. In the plane of the sagittal section, the vertical distance $(Z)$ was measured from the central point of specific MLNs to the upper edge of the sternum. The mean $\mathrm{X}, \mathrm{Y}$ and $\mathrm{Z}$ distances of specific MLNs were calculated as the drilling points of ROIs. The difference of $\mathrm{X}, \mathrm{Y}$ and $\mathrm{Z}$ distances between the male patients and female CDP were modified according to the proportion of Phantom products. Drilling on the CDP was conducted at Chengdu Phantom, Ltd. (Chengdu, China). 

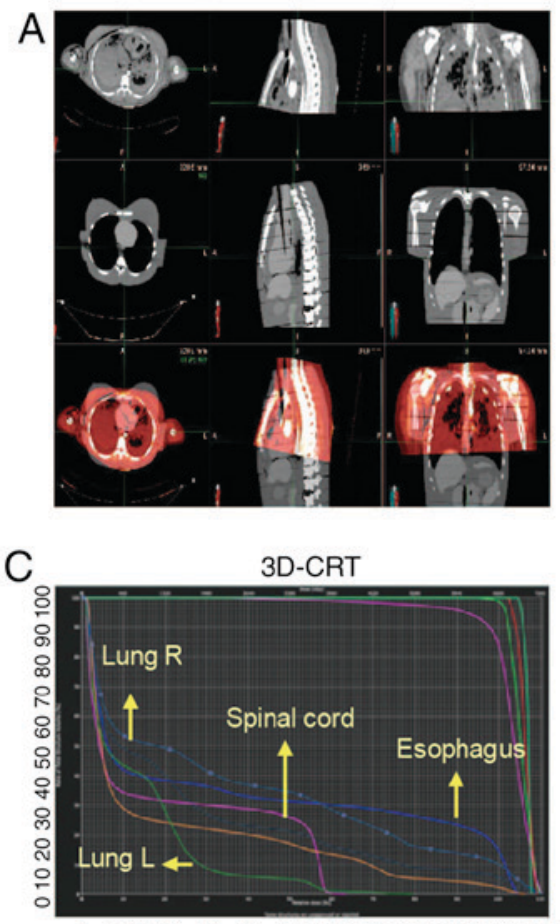

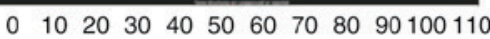

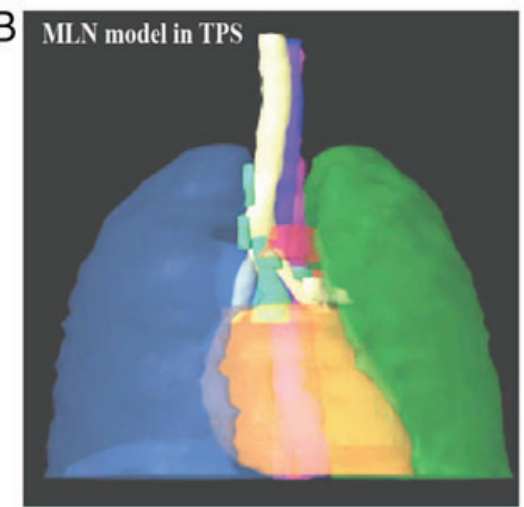

IMRT

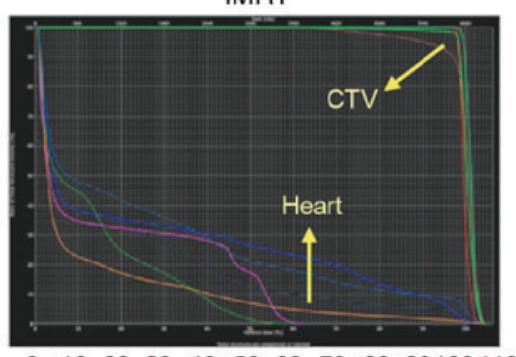

$0 \quad 102030405060708090100110$
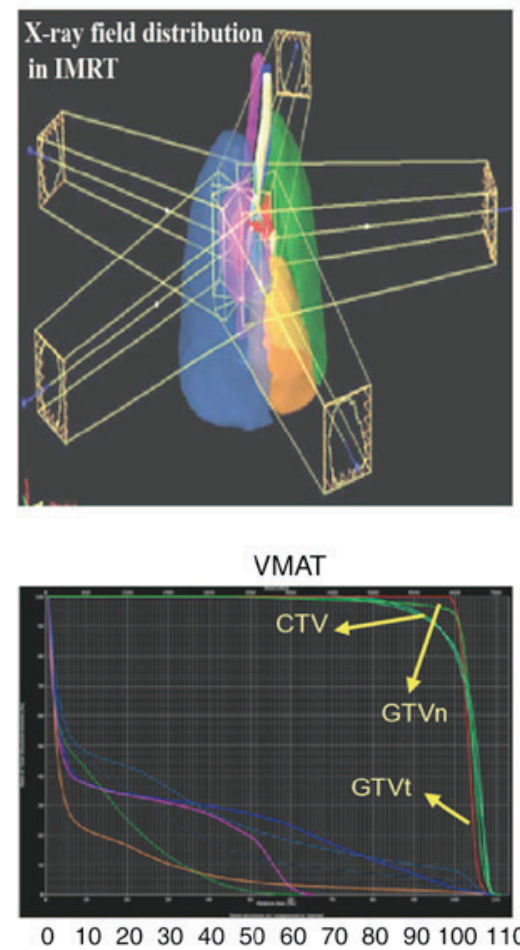

Figure 2. Image registration and fusion, and plan design for thoracic radiotherapy. (A) A 3D MLN model and computed tomography images registered and fused automatically in the Eclipse ${ }^{\mathrm{TM}}$ TPS. (B) A 3D MLN model in the Eclipse TPS. X-ray field distribution of IMRT as a representative. (C) The dose volume histogram of three plans in the fused MLN model (3D conformal radiotherapy, IMRT and VMAT). The same color lines in different panels represent the same regions of interest. MLN, mediastinal lymph node; CTV, clinical target volume; GTVn, MLNs at the 2nd, 4th and 7th stations; GTVt, gross tumor volume; IMRT, intensity-modulated radiotherapy; TPS, treatment planning system; 3D, three-dimensional; VMAT, volumetric-modulated arc therapy; 3D-CRT, three-dimensional conformal radiotherapy.

CT scan and plan design of CDP model. The CDP was scanned with a CT simulator (Philips Healthcare, Amsterdam, The Netherlands) with a slice thickness of $3 \mathrm{~mm}$. The Phantom CT images and DICOM format MLN images were uploaded to the MIM system (version 6.5.4; MIM Software Inc., Cleveland, $\mathrm{OH}, \mathrm{USA}$ ) of elastic deformation. In the image registration interface of the MIM system, two sets of images with the same position could be automatically registered and fused with rigid alignment. Multimodality fusion of primary and secondary images could be aligned automatically to correct possible differences in the $\mathrm{X}$-, Y- and Z-axes. The procedure was repeated three to five times to obtain the best fusion images. Fusion images were transferred into the Eclipse TPS. Contours of the SLL, MLNs and OARs were copied from 3D MLNs images to Phantom CT images for the plan design of 3D-CRT, IMRT and VMAT. The CTV concept was defined identical to that aforementioned. To keep the dosimetric measurement of the thermoluminescence dosimeter (TLD) in a linear range, CTV was dosed at 100 cGy irradiation by a 6-MV X-ray.

Measurement of radiation dose. Following the alignment of central points in the radiation field of the Phantom, any setup errors were corrected by cone beam CT, and then TLDs using lithium fluoride rods (3x10 mm; Chengdu Phantom Ltd) were plugged into drill points. TLDs are a type of lithium fluoride material, and the energy corrections and calibration were performed at the Center for Disease Control and Prevention of Sichuan (Chengdu, China). Additionally, the
Phantom underwent 100 cGy irradiation from the Trilogy ${ }^{\circledR}$ system linear accelerator (Varian Medical System, Inc.). The same procedures were conducted for all three plans. The dosimetric measurement of TLDs at 3 points of ROIs, including at the $2 \mathrm{R}$ and 7th MLN stations, and at SLL, was performed. All experiments were repeated three times.

Statistical analysis. All the data and readings of TLDs are presented as the mean \pm SD. Differences between groups were statistically analyzed using one-way analysis of variance (ANOVA) followed by a Student-Newman-Keuls post-hoc test and unpaired Student's t-test in SPSS version 18.0 (SPSS, Inc., Chicago, IL, USA). $\mathrm{P}<0.05$ was considered to indicate a statistically significant difference.

\section{Results}

$3 D$ reconstruction of $M L N s$ and adjacent structures. A 3D model of MLNs with surface and volume reconstructions was formed. The esophagus, trachea, left and right bronchi, thymus, sternum, thoracic duct and vertebrae, spinal cord, thoracic great vessels and heart were also reconstructed (Fig. 1C). The chest was divided into eight regions (11), which facilitated the identification of MLN stations (Fig. 1D and E). In the reconstructed 3D model of MLNs, MLN and adjacent structures were depicted, including front- and right-sided views (Fig. 1F and $\mathrm{G}$ ). Additionally, further details of adjacent structures were depicted, including the lungs, sternum, esophagus, trachea, spinal cord, heart and great vessels, with front- and left-sided 
Table I. Plan comparison of distinct radiotherapy technologies $(n=10)$.

P-value

\begin{tabular}{|c|c|c|c|c|c|c|}
\hline Parameters & $3 \mathrm{D}-\mathrm{CRT}^{\mathrm{a}}$ & IMRT $^{\mathrm{a}}$ & VMAT $^{\mathrm{a}}$ & 3D-CRT vs. IMRT & 3D-CRT vs. VMAT & IMRT vs. VMAT \\
\hline $\mathrm{D}_{2 \% \text { стV }}, \mathrm{cGy}$ & $7177.6 \pm 86.1$ & $7017.1 \pm 105.7$ & $7024.9 \pm 94.2$ & 0.001 & 0.001 & 0.863 \\
\hline $\mathrm{D}_{98 \% \mathrm{CTV}}, \mathrm{cGy}$ & $6609.2 \pm 135.4$ & $6452 \pm 33.7$ & $6501.4 \pm 38.2$ & 0.002 & 0.026 & 0.006 \\
\hline $\mathrm{D}_{\text {mean CTV }}, \mathrm{cGy}$ & $6925.6 \pm 52.3$ & $6838.1 \pm 64.5$ & $6826.8 \pm 61.4$ & 0.016 & 0.001 & 0.693 \\
\hline $\mathrm{V}_{5 \text { Double Lungs }}, \%$ & $46.52 \pm 6.5$ & $50.26 \pm 2.5$ & $58.78 \pm 4.8$ & 0.107 & 0.002 & 0.001 \\
\hline $\mathrm{V}_{20 \text { Double Lungs }}, \%$ & $28.46 \pm 4.6$ & $25.31 \pm 1.8$ & $28.26 \pm 2.4$ & 0.061 & 0.905 & 0.007 \\
\hline $\mathrm{V}_{30 \text { Double Lungs }}, \%$ & $23.69 \pm 3.2$ & $18.1 \pm 1.0$ & $18.99 \pm 0.6$ & $<0.001$ & 0.002 & 0.036 \\
\hline MLD, cGy & $1752.9 \pm 180.0$ & $1529.9 \pm 62.5$ & $1666.5 \pm 97.5$ & 0.001 & 0.198 & 0.001 \\
\hline $\mathrm{D}_{\text {Max Spinal Cord, }} \mathrm{cGy}$ & $3816.5 \pm 46.7$ & $3665.5 \pm 38.8$ & $3761 \pm 38.7$ & $<0.001$ & 0.009 & $<0.001$ \\
\hline $\mathrm{V}_{40 \text { Heart, }} \%$ & $28.6 \pm 10.9$ & $22.11 \pm 8.2$ & $12.32 \pm 7.8$ & 0.151 & 0.001 & 0.014 \\
\hline $\mathrm{CI}$ & $1.72 \pm 0.25$ & $1.07 \pm 0.08$ & $1.11 \pm 0.06$ & $<0.001$ & $<0.001$ & 0.241 \\
\hline $\mathrm{HI}$ & $1.10 \pm 0.03$ & $1.08 \pm 0.03$ & $1.09 \pm 0.03$ & 0.316 & 0.675 & 0.675 \\
\hline
\end{tabular}

${ }^{a}$ Mean \pm standard deviation. 3D-CRT, three-dimensional conformal radiotherapy; IMRT, intensity-modulated radiotherapy; VMAT, volumetric-modulated arc therapy; SD, standard deviation; CTV, clinical target volume; MLD, mean lung dose; D, dose; V, volume; CI, conformity index; HI, homogeneity index.

views (Fig. 1H and I). The 9th MLN station and other groups of the ITLN were not identified in CVH2.

Plan comparison in different radiotherapy technologies. Following image registration and fusion in Eclipse TPS, a successful match was obtained of the mediastinum, lungs, backbone and OARs between the 3D MLN model and chest CT (Fig. 2A). In radiotherapy, contouring of MLNs is generally based on the CT image set. The 3D MLN model with high-resolution fusion photography could optimize the MLN contours. The cadaver data for target delineation were much clearer than chest CT, even if contrast agents were used in CT or MRI imaging, due to the blood vessel perfusion imaging and high resolution $(15,16)$. The MLN model can improve the clarity and resolution of CT images notably (Fig. 2B). The treatment plans of 3D-CRT, IMRT and VMAT were designed in the Eclipse TPS.

The dose of 2 percent target volume $\left(\mathrm{D}_{2 \%}\right)$, dose of 98 percent target volume $\left(\mathrm{D}_{98 \%}\right)$ and mean dose of target volume $\left(\mathrm{D}_{\text {mean }}\right)$ of CTV in 3D-CRT were increased compared with those in IMRT and VMAT. Compared with IMRT, there were no differences in $\mathrm{D}_{2 \%}$ and $\mathrm{D}_{\text {mean }}$ of CTV in VMAT, but differences were identified in $\mathrm{D}_{98 \%}$. The volume of $20 \mathrm{~Gy}\left(\mathrm{~V}_{20}\right)$, volume of $30 \mathrm{~Gy}\left(\mathrm{~V}_{30}\right)$ and mean lung dose (MLD) of the lungs in IMRT were decreased compared with those in 3D-CRT and VMAT. The $\mathrm{V}_{5}$ of the lungs in VMAT was higher than that in 3D-CRT and IMRT. The maximum dose of the spinal cord $\left(\mathrm{D}_{\mathrm{Max}}\right)$ was lowest in IMRT, and the heart dose $\left(\mathrm{V}_{40}\right)$ was lowest in VMRT.

Conformity index $\left(\mathrm{CI} ; \mathrm{CI}=\mathrm{V}_{\mathrm{RI}} / \mathrm{V}_{\mathrm{TV}}\right)$ and homogeneity index $\left(\mathrm{HI} ; \mathrm{HI}=\mathrm{D}_{\mathrm{Max}} / \mathrm{RI}\right)$ were also calculated as per The Radiation Therapy Oncology Group definition (26,27). In the present study, RI was the prescribed dose of PTV, $\mathrm{V}_{\mathrm{RI}}$ was the volume of the RI, $\mathrm{V}_{\mathrm{TV}}$ was the total volume of PTV and $\mathrm{D}_{\text {Max }}$ was the maximum dose. The CI in IMRT and VMAT was better than that in 3D-CRT, but there was no difference in HI among the three radiotherapy plans. All these comparisons were significantly different $(\mathrm{P}<0.05$; Table I; Fig. 2C). Taken in combination, IMRT demonstrated significantly higher values in CI, CTV coverage and OARs (lungs and spinal cord) protection compared with 3D-CRT and VMAT $(\mathrm{P}<0.05)$.

Location of MLNs and drilling the radiation phantom. Among the 20 male patients with thoracic tumors in Oncology Department, Xinqiao Hospital (Chongqing, China), there were 9 cases of lung squamous cell carcinoma, 1 case of lung adenosquamous carcinoma, 7 cases of small cell lung cancer, 1 case of esophageal carcinoma and 2 cases of other tumor types. The physical characteristics were as follows: Median age, 57 years; median height, $169.4 \mathrm{~cm}$; median weight, $66.6 \mathrm{~kg}$; and median body surface area, $1.80 \mathrm{~m}^{2}$. The vertical distances X (Fig. 3A), Y (Fig. 3B) and Z (Fig. 3C) of the 2R, 2L, 4R, 4L and 7th MLN stations were measured from their chest CT images (data not shown) and the mean distances were calculated. The MLN ROIs were then drilled into the CDP model (Figs. 3D-F). The drill points were $3 \times 10 \mathrm{~mm}$ in size, which matched the TLDs.

Dosimetric measurement in distinct radiotherapy plans. The chest CT scan of the CDP demonstrated that each drill point was clearly located in specific MLN stations. A simulation model of the MLNs for thoracic radiotherapy was then established (Fig. 4A). Since dosimetric variation existed between actual absorbed and calculated doses, the same dosimetric measurement was repeated among the 3D-CRT, IMRT and VMAT plans, and the dosimetric variation in different ROIs was identified. In DVH, the doses (mean \pm SD) of CTV at 3 points were calculated ( $2 \mathrm{R}$ and 7 th MLN stations, and SLL) (Table II; Fig. 4B-D) and the dosimetric measurement of TLDs was made for the three distinct radiotherapy technologies. Through statistical analysis, bias was identified between measured and calculated doses in three distinct 

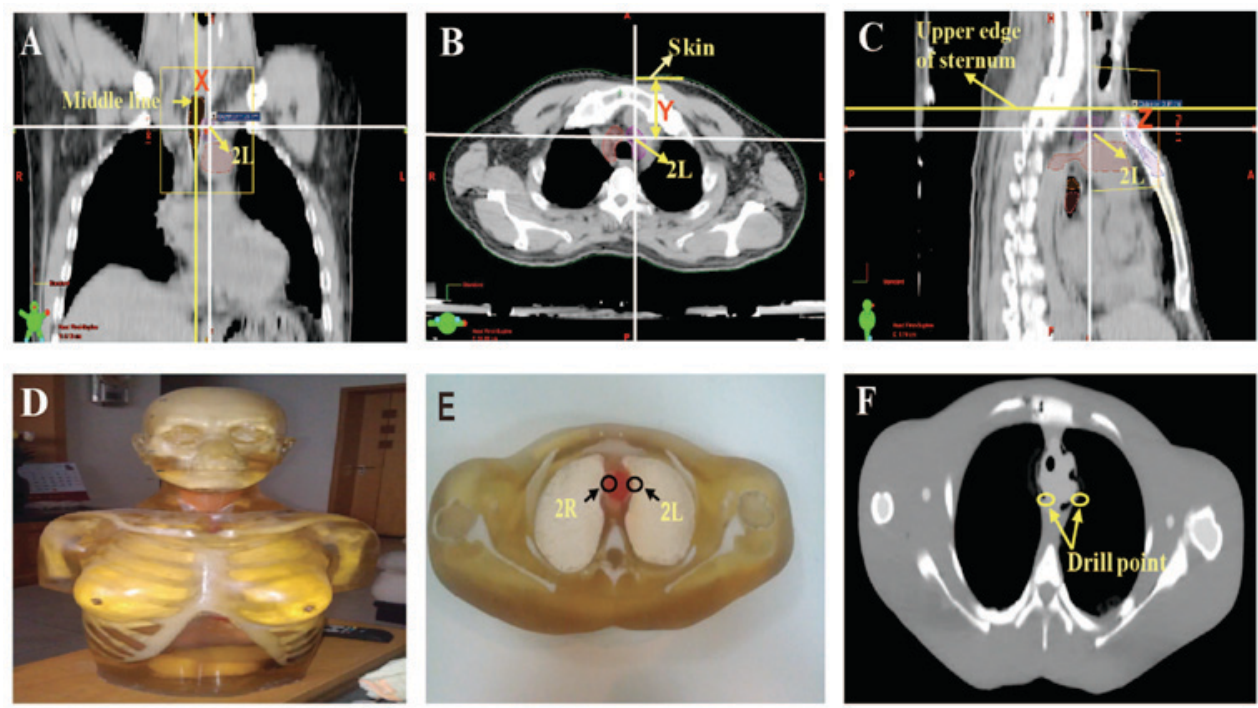

Figure 3. Drill points on the CDP. (A) The vertical distance $\mathrm{X}$ from the central point of the $2 \mathrm{~L}$ region to the middle line in the coronal-sectional plane. (B) The vertical distance $\mathrm{Y}$ from the central point of the $2 \mathrm{~L}$ region to the surface skin in the transverse-sectional plane. (C) The vertical distance $\mathrm{Z}$ from the central point of the $2 \mathrm{~L}$ region to the upper edge of the manubrium sterni in the sagittal-sectional plane. (D) A female CDP model with head, neck and chest. (E) Drill points of the $2 \mathrm{R}$ and $2 \mathrm{~L}$ stations at CDP. (F) Drill points of the $2 \mathrm{R}$ and $2 \mathrm{~L}$ stations on CDP computed tomography image. CDP, Chengdu Dosimetric Phantom.
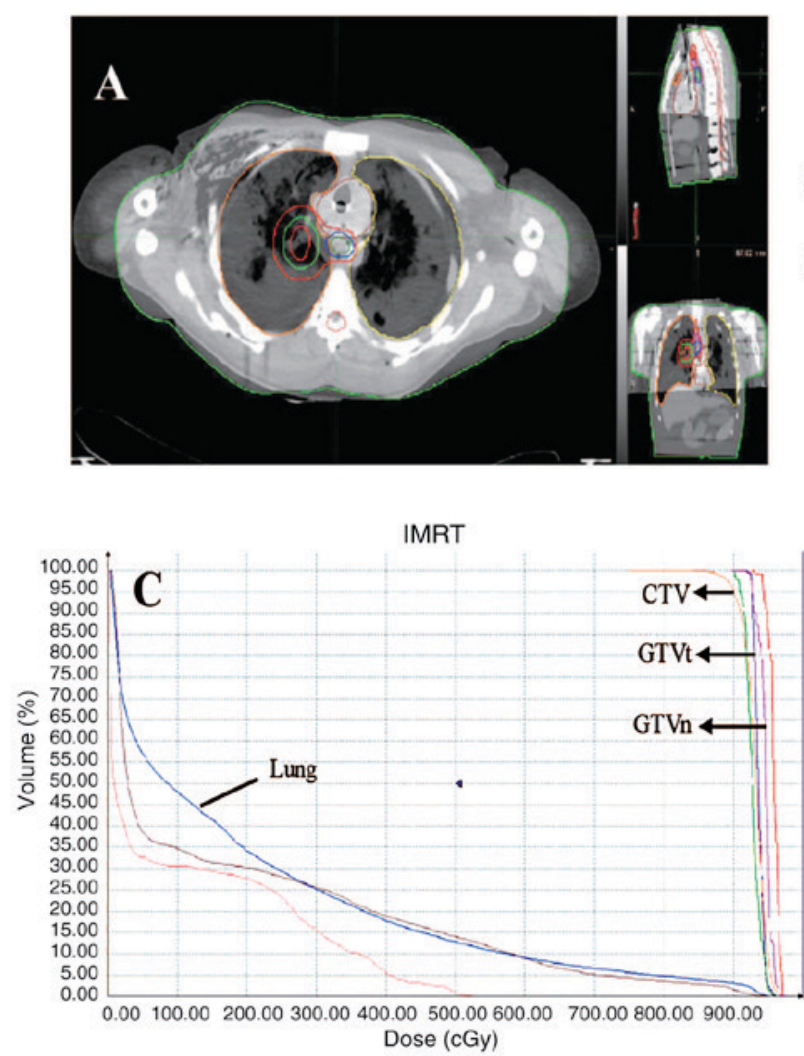
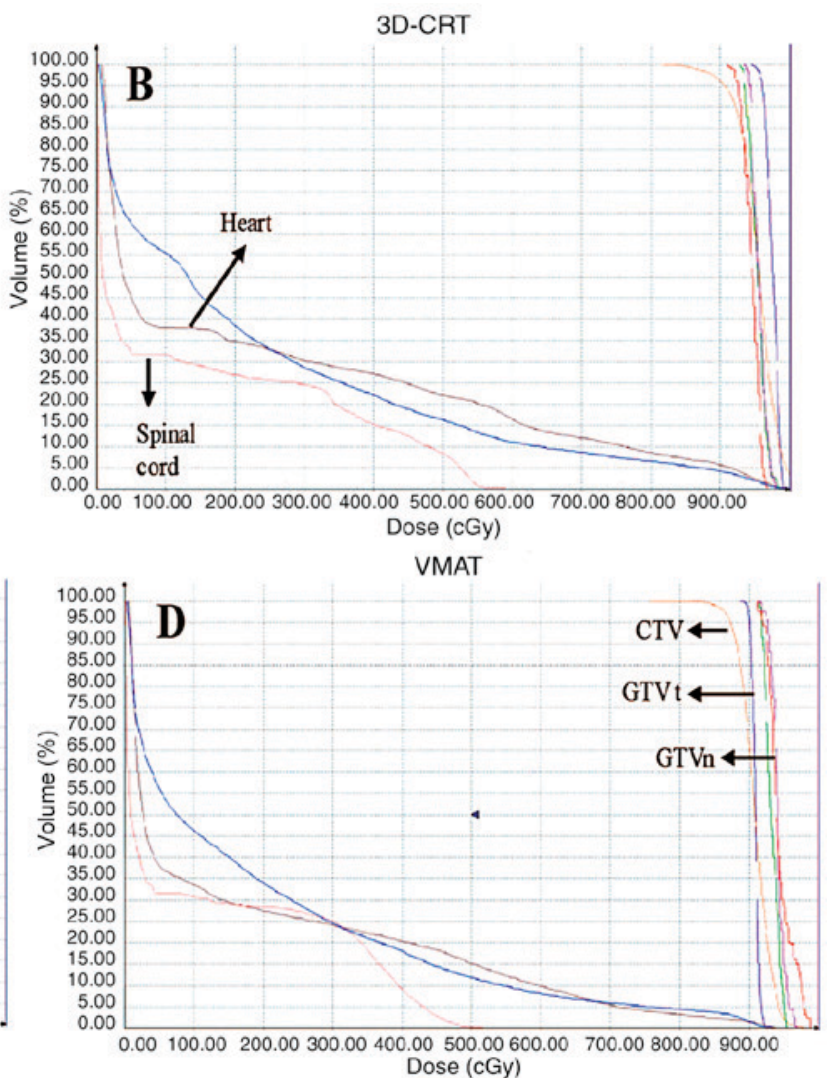

Figure 4. Contours and DVH of three radiotherapy plans in the MLN model. (A) Contours of MLNs, simulated lung lesion and organs at risk on fused images in the MIM system. (B) DVH of the 3D-CRT plan. (C) DVH of the IMRT plan. (D) DVH of the VMAT plan. The same color lines in different panels represent the same regions of interest. Note that the arrow for GTVn is referring to the purple line in both (C) and (D) graphs. DVH, dose-volume histogram; MLN, mediastinal lymph node; 3D-CRT, three-dimensional conformal radiotherapy; IMRT, intensity-modulated radiotherapy; VMAT, volumetric-modulated arc therapy; CTV, clinical target volume; GTVn, MLNs at the 2nd, 4th and 7th stations; GTVt, gross tumor volume.

plans (Table II). In combination, the measurement doses were within $\pm 10 \%$ deviation, compared with calculated doses at SLL, and at $2 \mathrm{R}$ and 7 th MLN stations, indicating dosimetric bias in the calculated doses (Table II).

\section{Discussion}

In thoracic malignancy, tumor, node, metastasis (TNM) staging serves an important role in selecting the treatment options and 


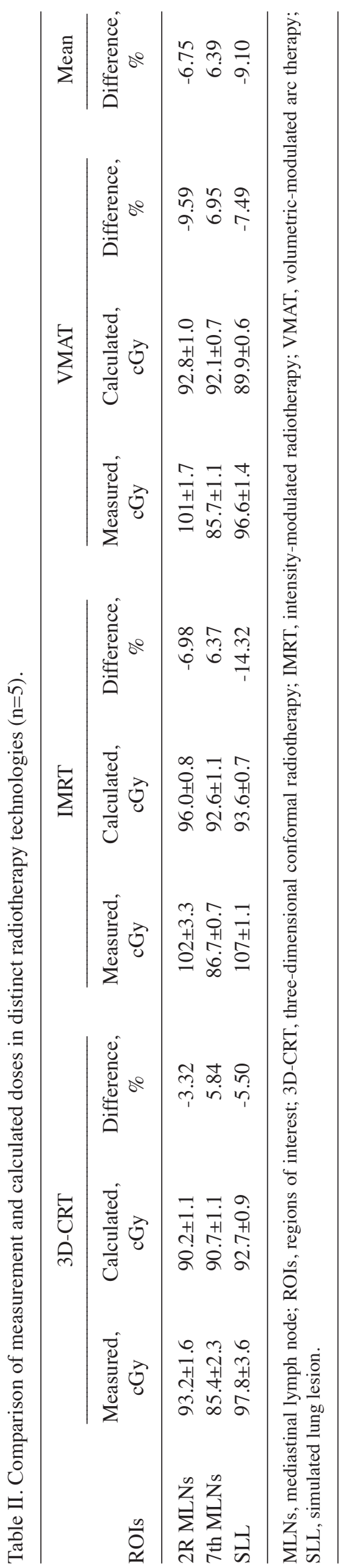

calculating the prognosis estimation (28). Weder (29) identified that the TNM staging system was a vital reference for treatment and prognostic analysis in thoracic malignancy. In the present study, the 3D MLN model, observed by unaided eyes, was superior to the CT and MRI images in terms of high definition, resolution and recognition. Although a previous study reported reconstruction of thoracic structures (17), to the best of our knowledge, the present study is the first to construct a 3D MLN model based on the CVH dataset. This model is a useful teaching tool, and it can provide morphological data for imaging diagnosis, facilitating a self-learning approach for the chest anatomy for medical students and for navigating the thoracic cavity during surgery for junior surgeons. The visualized MLN model can provide additional valuable information and assist in the understanding of miniscule and spatial structures, arousing a Student's interest in anatomy and overcoming the teaching difficulty when there is a lack of human cadavers; however, this function of the 3D MLN model is not the focus of the present study and will not be discussed further.

The 3D MLN model can also function as a contouring aid. The user can view single or multiple contours rendered on the anatomical or CT scan images for CVH2; thus, the MLN model allows the user to practice the contouring process of anatomical structures and improve their ability to contour MLNs. The 3D MLN digital model was used in combination with the chest CT, which is based on a medium-build figure, to ensure uniform conditions in the present study and prevent deviation due to the diversity of patients. Image fusion between the 3D MLN model and chest CT was performed; thus, it was easier to recognize MLNs and OARs on fused images. There is also a possibility to simulate thoracic radiotherapy in TPS and even dynamically visualize radiation fields from different directions and angles in coplanar radiation. Based on the standard evaluation model, a definite dosimetric deviation and radiation plan can be determined for different thoracic radiotherapy strategies. Without a 3D MLN model combined with the standard chest CT images, a group of patients would have been required to compare different plans, which would have raised the issue of avoiding interpatient diversity and uncertain MLN contours.

Furthermore, the 3D MLN model can be used for dosimetric measurement in thoracic radiotherapy supported by CDP, a humanoid-shaped bioequivalent material of Chinese individuals. Since the CDP is based on a Chinese patient of a medium-build, it ensured uniform conditions and prevented interpatient deviation in the present study. The majority of commercially available dosimetric devices in quality assurance, including the solid water and delta 4 device, have homogeneous density throughout their volume (30). There are also certain heterogeneous phantoms, including the Rando ${ }^{\circledR}$ phantom (31). By contrast, CDP is an inhomogeneous phantom that has variable and varying densities inside, similar to that of the real interior of a body. Therefore, it could achieve a precise radiotherapy target volume, positioning and release dose using modern radiation technology. Peng et al (25) verified CDP as a good avatar for humans via studying the tissue equivalent imaging. Comparisons between the $\mathrm{CT}$ values of the CDP and humans demonstrated a deviation of $<5 \%$ (25). The current study is based on these earlier reports, and brings CDP into preclinical research. 
Eclipse TPS supports image registration and fusion of the 3D MLN model and CDP CT images. Thus, CDP can be radiation detector simulator with the use of TLDs. In the present study, four sets of data of TLDs were tested, with the X-ray at 25, 50, 75 and 100 cGy, respectively. The measurement value linearly increased with the increasing X-ray radiation (data not shown), indicating that TLDs are an ideal tool to measure the actual dose of the radiation phantom.

The current study demonstrated that different radiation technologies have different dose-volume effects. IMRT possessed improved $\mathrm{V}_{20}, \mathrm{~V}_{30}$ and MLD of the lungs with similar CTV coverage. Furthermore, the present study indicated that there are dosimetric deviations between measured and calculated doses. To the best of our knowledge, the current study was the first to combine the 3D MLN model with the CDP model to optimize the plan of thoracic radiotherapy. This newly built MLN model could be an important tool in improving the effectiveness of thoracic radiotherapy in future clinical studies. Similarly, the present study lays the foundation for other ROI studies in dosimetric phantoms and sets an example for radiotherapy studies of other cancer types.

There is significant dose variation in the soft tissue/skin, solid tissue/lung and soft tissue/bone, due to the backscatter radiation from the interface in the treatment region (32). Although modern TPS is believed to have the ability to successfully predict the dose that should be given to the patient, the actual absorbed doses generally deviate from calculated doses in practice. In a study of stereotactic lung radiotherapy, two heterogeneous phantoms were conducted with targets of 1.5 and $4.0 \mathrm{~cm}$. Dose distributions in the simulated tumors delivered by different treatment plans were measured with radiochromic film. The dosimetric inaccuracy ranged from -3 to $4 \%$ (33). In another study of dose distribution for VMAT applied to total marrow irradiation in a human-like phantom, readings of TLDs demonstrated a dose difference from -4.3 to $6.6 \%$ compared with the calculated dose (34). To examine if there were variations in different radiotherapy strategies, the dosimetric measurement was repeated among the 3D-CRT, IMRT and VMAT plans in the present study. Based on the standard evaluation model of CDP, a definite dosimetric deviation can be determined for different thoracic radiotherapy strategies, thus avoiding interpatient diversity and uncertain MLNs contour. The deviation at SLL, and at the $2 \mathrm{R}$ and 7th MLN stations was identified. A larger deviation was demonstrated between measured and calculated doses, which may have been caused by the different CTVs and linear accelerators. Collectively, all these studies indicated that it is necessary to apply dosimetric phantoms in clinical dosimetric monitoring.

There are certain limitations to the present study. Firstly, the CDP was produced based on selected male patients of a medium-build, due to the female patients being shorter and thinner than the medium figure. Additionally, the present study was not rigorous enough to design radiotherapy plans and measure the dose in the same CDP. In addition, visual models were insufficient for individualized radiotherapy plans of real-world patients. The depth of thoracic cavity in the prone posture serves a critical role in dose deposition, which means that the individualized radiotherapy plans should be optimized with compensation of bioequivalent material of CDP to realize an adjustable thickness. Furthermore, a set of criteria is also required for using and evaluating 3D MLN models and radiation phantoms in thoracic radiotherapy. More detailed studies are required to further confirm the conclusions. Since MIM has the function of deformable image registration in internal structures between MLNs images and chest CT, MIM and deformable registration could be used to extend this model to other patients using an atlas-based approach.

In the future, a 3D movable virtual system or 4D application could be used in the future for dosimetric monitoring of novel radiotherapy techniques, including breath gating or target tracking treatment, since 4D-radiotherapy is gaining popularity (35).

In conclusion, clinical diagnosis and treatment may greatly benefit from digital medical models for radiotherapy. The 3D MLN model in the present study can benefit plan optimization and dosimetric measurement of thoracic radiotherapy, and when combined with CDP, it may provide a tool for clinical dosimetric monitoring.

\section{Acknowledgements}

The authors are grateful to The National Key Research and Development Project (grant no. 2016YFC0106400), The National Natural Science Foundation of China (grant no. 81272496), The Natural Science Foundation of Chongqing (grant nos. cstc2012jjA10096 and cstc2013kjrc-tdjs10011) and The Clinical Foundation of Third Military Medical University (grant no. 2011XLC46) for financial support. In addition, the authors would like to thank Professor Da-Quan Lin at Sichuan University (Chengdu, Sichuan, China) for guidance in drilling the CDP and in the dosimetric measurement of TLDs.

\section{References}

1. Jemal A, Bray F, Center MM, Ferlay J, Ward E and Forman D: Global cancer statistics. CA Cancer J Clin 61: 69-90, 2011.

2. Allemani C, Weir HK, Carreira H, Harewood R, Spika D, Wang XS, Bannon F, Ahn JV, Johnson CJ, Bonaventure A, et al: Global surveillance of cancer survival 1995-2009: Analysis of individual data for $25,676,887$ patients from 279 population-based registries in 67 countries (CONCORD-2). Lancet 385: 977-1010, 2015.

3. Diwanji TP,Mohindra P, Vyfhuis M, Snider JW III,KalavaguntaC, Mossahebi S, Yu J, Feigenberg S and Badiyan SN: Advances in radiotherapy techniques and delivery for non-small cell lung cancer: Benefits of intensity-modulated radiation therapy, proton therapy, and stereotactic body radiation therapy. Transl Lung Cancer Res 6: 131-147, 2017.

4. Jensen I, Carl J, Lund B, Larsen EH and Nielsen J: Radiobiological impact of reduced margins and treatment technique for prostate cancer in terms of tumor control probability (TCP) and normal tissue complication probability (NTCP). Med Dosim 36: 130-137, 2011.

5. Palma DA, Verbakel WF, Otto K and Senan S: New developments in arc radiation therapy: A Review. Cancer Treat Rev 36: 393-399, 2010.

6. Chan OS, Lee MC, Hung AW, Chang AT, Yeung RM and Lee AW: The superiority of hybrid-volumetric arc therapy (VMAT) technique over double arcs VMAT and 3D-conformal technique in the treatment of locally advanced non-small cell lung cancer-A planning study. Radiother Oncol 101: 298-302, 2011.

7. Rauschenbach BM, Mackowiak L and Malhotra HK: A dosimetric comparison of three-dimensional conformal radiotherapy, volumetric-modulated arc therapy, and dynamic conformal arc therapy in the treatment of non-small cell lung cancer using stereotactic body radiotherapy. J Appl Clin Med Phys 15: 4898, 2014. 
8. Wu Z, Xie C, Hu M, Han C, Yi J, Zhou Y, Yuan H and Jin X: Dosimetric benefits of IMRT and VMAT in the treatment of middle thoracic esophageal cancer: Is the conformal radiotherapy still an alternative option? J Appl Clin Med Phys 15: 93-101, 2014.

9. Bzdusek K, Friberger H, Eriksson K, Hårdemark B, Robinson D and Kaus M: Development and evaluation of an efficient approach to volumetric arc therapy planning. Med Phys 36: 2328-2339, 2009.

10. Heineman DJ, Daniels JM and Schreurs WH: Clinical staging of NSCLC: Current evidence and implications for adjuvant chemotherapy. Ther Adv Med Oncol 9: 599-609, 2017.

11. Mountain CF and Dresler CM: Regional lymph node classification for lung cancer staging. Chest 111: 1718-1723, 1997.

12. Rusch VW, Asamura H, Watanabe H, Giroux DJ, Rami-Porta R and Goldstraw P; Members of IASLC Staging Committee: The IASLC lung cancer staging project: A proposal for a new international lymph node map in the forthcoming seventh edition of the TNM classification for lung cancer. J Thorac Oncol 4: 568-577, 2009.

13. Lababede O, Meziane M and Rice T: Seventh edition of the cancer staging manual and stage grouping of lung cancer: Quick reference chart and diagrams. Chest 139: 183-189, 2011.

14. Shen G, Lan Y, Zhang K, Ren P and Jia Z: Comparison of 18F-FDG PET/CT and DWI for detection of mediastinal nodal metastasis in non-small cell lung cancer: A meta-analysis. PLoS One 12: e0173104, 2017.

15. Zhang SX, Heng PA, Liu ZJ, Tan LW, Qiu MG, Li QY, Liao RX, Li K, Cui GY, Guo YL, et al: Creation of the Chinese visible human data set. Anat Rec B New Anat 275: 190-195, 2003.

16. Zhang SX, Heng PA, Liu ZJ, Tan LW, Qiu MG, Li QY, Liao RX, Li K, Cui GY, Guo YL, et al: The Chinese Visible Human (CVH) datasets incorporate technical and imaging advances on earlier digital human. J Anat 204: 165-173, 2004.

17. Wu Y, Luo N, Tan L, Fang B, Li Y, Xie B, Liu K, Chu C, Li M and Zhang S: Three-dimensional reconstruction of thoracic structures: Based on chinese visible human. Comput and Math Methods Med 2013: 795650, 2013

18. Rong J, Wang Q, Liu K, Tan L, Ran X, Zhang S, Li Q and Han Y: A new atlas localization approach for subthalamic nucleus utilizing Chinese visible human head datasets. PLoS One 8: e57264, 2013

19. Capelle L, Warkentin H, Mackenzie M, Joseph K, Gabos Z, Pervez N, Tankel K, Chafe S, Amanie J, Ghosh S, et al: Skin-sparing helical tomotherapy vs. 3D-conformal radiotherapy for adjuvant breast radiotherapy: In Vivo, skin dosimetry study. Int J Radiat Oncol Biol Phys 83: e583-e590, 2012.

20. Nazemi-Gelyan H, Hasanzadeh H, Makhdumi Y, Abdollahi S, Akbari F, Varshoee-Tabrizi F, Almasrou H, Nikoofar A and Rezaei-Tavirani M: Evaluation of organs at risk's dose in external radiotherapy of brain tumors. Iran J Cancer Prev 8: 47-52, 2015.

21. Adolfsson E, Gustafsson H, Lund E, Alm Carlsson G, Olsson S and Carlsson Tedgren S: A system for remote dosimetry audit of 3D-CRT, IMRT and VMAT based on lithium formate dosimetry. Radiother Oncol 113: 279-282, 2014.

22. Jiang X, Li T, Liu Y, Zhou L, Xu Y,Zhou X and Gong Y: Planning analysis for locally advanced lung cancer: Dosimetric and efficiency comparisons between intensity-modulated radiotherapy (IMRT), single-arc/partial-arc volumetric modulated arc therapy (SA/PA-VMAT). Radiat Oncol 6: 140, 2011.
23. ICRU report 48, phantoms and computational models in therapy, diagnosis and protection, 1992.

24. Lehmann J, Stern RL, Levy J, Daly TP, Siantar CL and Goldberg Z: Radiation phantom with humanoid shape and adjustable thickness (RPHAT). Phys Med Biol 49: N125-N129, 2004.

25. Peng G, Zeng Y, Luo T, Zhao F, Peng S, You R, Tan H, Liu X and Wang J: Organ dose evaluation for multi-slice spiral CT scans based on China Sichuan chest anthropomorphic phantom measurements. Radiat Prot Dosimetry 150: 292-297, 2012.

26. Feuvret L, Noël G, Mazeron JJ and Bey P: Conformity index: A review. Int J Radiat Oncol Biol Phys 64: 333-342, 2006.

27. Ayata HB, Güden M, Ceylan C, Kücük N and Engin K: Comparison of dose distributions and organs at risk (OAR) doses in conventional tangential technique (CTT) and IMRT plans with different numbers of beam in left-sided breast cancer. Rep Pract Oncol Radiother 16: 95-102, 2011.

28. Mountain CF: Revisions in the international system for staging lung cancer. Chest 111: 1710-1717, 1997.

29. Weder W: Lung cancer: New opportunities-changing algorithm in staging. Ann Oncol 19 (Suppl 7): vii28-vii30, 2008.

30. Gurjar OP, Mishra SP, Bhandari V, Pathak P, Patel P and Shrivastav G: Radiation dose verification using real tissue phantom in modern radiotherapy techniques. J Med Phys 39: 44-49, 2014.

31. Yamazaki H, Iwama K, Nishimura T, Iwai Y, Aibe N, Takenaka T, Miyake S, Tanaka E, Yoshida K, Oota Y, et al: Comparison of calculated dose by helical tomotherapy treatment planning machine and measured dose of radiophotoluminescence glass dosimeter in lung lesions using Rando Phantom. Anticancer Res 33: 1679-1684, 2013.

32. Kinhikar RA, Tambe CM, Patil K, Mandavkar M, Deshpande DD, Gujjalanavar R, Yadav P and Budrukkar A: Estimation of dose enhancement to soft tissue due to backscatter radiation near metal interfaces during head and neck radiothearpy-A phantom dosimetric study with radiochromic film. J Med Phys 39: 40-43, 2014.

33. Seppala J, Suilamo S, Kulmala J, Mali P and Minn H: A dosimetric phantom study of dose accuracy and build-up effects using IMRT and RapidArc in stereotactic irradiation of lung tumours. Radiat Oncol 7: 79, 2012

34. Surucu M, Yeginer M, Kavak GO, Fan J, Radosevich JA and Aydogan B: Verification of dose distribution for volumetric modulated arc therapy total marrow irradiation in a humanlike phantom. Med Phys 39: 281-288, 2012.

35. Padmanaban S, Boopathy R, Kunjithapatham B, Sukumar P and Nagarajan V: A phantom study on the effects of target motion in non-gated kV-CBCT imaging. Australas Phys Eng Sci Med 33: 59-64, 2010.

This work is licensed under a Creative Commons Attribution-NonCommercial-NoDerivatives 4.0 International (CC BY-NC-ND 4.0) License. 